\title{
LA REGULACIÓN DE LA OFERTA DE CARRERAS DESDE LA PERSPECTIVA DE UNA UNIVERSIDAD PRIVADA AUTÓNOMA
}

Roberto Guerrero* 


\section{RESUMEN}

El artículo enfoca el tema de la regulación de oferta de carreras universitarias desde el punto de vista de una universidad privada autónoma. Para estos efectos hace referencia, en primer término, al origen de las nuevas universidades privadas y su papel en la sociedad. Luego analiza el tema de la demanda de educación universitaria, para enseguida esbozar cómo una universidad privada adopta las decisiones en materia de oferta de carreras.

A continuación, aborda el aspecto más polémico: la posibilidad de que terceros regulen esta oferta, desde un ámbito cuantitativo y cualitativo. Por último, el artículo enfatiza como tema de fondo la necesidad de una adecuada información en esta materia.

\section{ABSTRACT}

The author approaches the issue of regulating the supply of university degree programs from the point of view of a private, independent university. He first addresses the subject of the origin of private universities and the role they play in society. Then he analyzes the issue of the demand for university education and goes on to outline how a private university makes decisions in terms of the degree programs it offers.

Guerrero then addresses the most controversial aspect: the possibility of having third parties regulate the supply, in quantitative and qualitative terms. Finally, the author outlines his own views on the subject and emphasizes the crucial need for adequate information on this matter. 


\section{LA REGULACIÓN DE OFERTA DE CARRERAS DESDE LA PERSPECTIVA DE UNA UNIVERSIDAD PRIVADA AUTÓNOMA}

\section{INTRODUCCIÓN}

Para una adecuada comprensión del enfoque que adopta una universidad privada autónoma en la determinación de oferta de carreras universitarias, me parece que es indispensable hacer una referencia, a lo menos sucinta, al origen de estas instituciones y a su regulación particular.

Como se recordará, ellas surgen por efecto del DFL $N^{\circ} 1$, de 30 de diciembre de 1980, el cual, además de definir lo que se entiende por universidades y sus fines; de fijar el concepto de autonomía universitaria y libertad académica y de determinar los grados académicos y títulos profesionales que estas instituciones pueden conferir, contempla la creación y disolución de universidades, con un mecanismo que introduce la posibilidad de constituir, para estos efectos, nuevas entidades como personas jurídicas de derecho privado sin fines de lucro.

Aun cuando en el estatuto jurídico antes referido no hay considerandos que expliciten los fundamentos de esta normativa, ni del contexto histórico en que ella se dictó y de la realidad universitaria del momento, es posible identificar algunas razones que promovieron esta reglamentación y que dieron lugar al surgimiento de universidades privadas:

1) En esa época la oferta de carreras universitarias era bastante limitada en relación con una creciente demanda; 2) no era factible que el Estado concurriera a un mayor financiamiento directo de las universidades históricas o tradicionales, de manera que aplicando el principio de la subsidariedad era conveniente permitir la creación de entidades que, sin recurrir al financiamiento estatal, hicieran posible aumentar la oferta universitaria; 3) la necesidad de introducir una saludable 
competencia en el ámbito universitario, y aumentar la libertad personal en dicha área, de modo de terminar con el monopolio estatal en este aspecto de la enseñanza.

El aludido cuerpo legal no contempló normas especiales sobre la forma en que las nuevas universidades podían ofrecer carreras, de manera que debió entenderse que ella quedó al arbitrio de las mismas, obviamente ateniéndose a las disposiciones de la normativa en cuestión. En esos comienzos, la principal limitación a la oferta estaba más bien determinada por la aprobación de los respectivos programas de estudios por parte de la correspondiente universidad examinadora.

Aun cuando en teoría ello hubiera permitido un crecimiento muy alto en la oferta de carreras universitarias, lo anterior no ocurrió por cuanto en una primera etapa sólo se constituyeron tres universidades privadas, atendidas las restricciones derivadas de la situación económica general del país en la época y de la modificación operada en el financiamiento indirecto de las universidades. Esta situación implicó una readecuación de los proyectos de nuevas entidades y en muchos casos, entre ellos el de la Universidad Finis Terrae, la postergación de los mismos.

Posteriormente surge la Ley Orgánica Constitucional de Enseñanza, No 18.962, de 10 de marzo de 1990, que crea el Consejo Superior de Educación (CSE) y que contiene nuevas regulaciones que inciden de manera importante sobre la forma de realizar la oferta de carreras, introduciendo todo el proceso de acreditación, entendido éste en la forma en que la ley lo regula y no como se entiende en la actualidad.

De todos modos, a través de tal proceso de acreditación, el CSE entra a velar por la forma en que puede surgir la oferta de carreras universitarias. No obstante, es preciso reconocer que no se introducen limitaciones a la oferta cuantitativa de carreras, sino que se apunta al aspecto cualitativo, vinculado estrechamente a la capacidad para impartir una determinada carrera, contando con los planteles docentes calificados, infraestructura adecuada y elementos básicos, especialmente bibliotecológicos, que permitan impartir la carrera en debida forma. 
Tanto el primitivo DFL $\mathrm{N}^{\circ} 1$ como la actual Ley $\mathrm{N}^{\circ} 18.962$, reconocen explícitamente la autonomía de los establecimientos de educación superior, que según define dicha ley es "el derecho de cada establecimiento a regirse por sí mismo, de conformidad con lo establecido en sus estatutos, en todo lo concerniente al cumplimiento de sus finalidades", comprendiendo a las autonomías académica, económica y administrativa. La disposición agrega que "La autonomía académica incluye la potestad de las entidades de educación superior para decidir por sí mismas la forma cómo se cumplan sus funciones de docencia, investigación y extensión y la fijación de sus planes y programas de estudios"1.

La preceptiva en cuestión destaca que "la libertad académica incluye la de abrir, organizar y mantener establecimientos educacionales, cumpliendo los requisitos establecidos por la ley, y la de buscar y enseñar la verdad conforme con los cánones de la razón y los métodos de la ciencia"².

En consecuencia, el ejercicio de la autonomía, entendido como ejercicio responsable, es el que provee el marco dentro del cual las universidades privadas autónomas pueden realizar y determinar la oferta de carreras universitarias.

\section{LA DEMANDA POR EDUCACIÓN Y POR CARRERAS UNIVERSITARIAS}

Es de la esencia de toda universidad el cultivo de las Ciencias y de las Artes, que se traduce en una divulgación del saber y en tareas de investigación destinadas a ahondar el conocimiento y descubrir nuevos aspectos del mismo. La universidad es, además, una institución que surge en y para un medio social y que tiene por lo mismo una responsabilidad para con la colectividad donde se encuentra inserta ${ }^{3}$.

La tarea de una universidad no es entonces realizar ingeniería social, es decir, pretender estructurar la sociedad sobre la base de un

1. Véase bibliografía final.

2. Véase bibliografía final.

3. Véase bibliografía final. 
modelo predefinido, sino dar satisfacción a lo que la sociedad requiere en el aspecto específico de su ámbito de acción, que ya hemos definido como la búsqueda y transmisión del conocimiento.

Es determinante entonces que las universidades, al resolver y estudiar el desarrollo de una determinada carrera, tengan en consideración la respectiva demanda existente en el medio social, puesto que así podrán satisfacer una necesidad que el cuerpo social está requiriendo. En este sentido, la demanda por educación en nuestra sociedad es creciente.

Nuestro mundo, el de comienzos del siglo XXI, es un mundo que está esencialmente en evolución, en el cual los cambios se producen con una vertiginosidad abrumadora, exigiendo de las nuevas generaciones que se incorporen a la vida laboral con un grado cada vez mayor de preparación y conocimientos, necesarios para entender la realidad y ser capaces de insertarse y participar en ella sin quedar excluidos.

En consecuencia, la globalización de nuestra cultura actual y la necesidad de progreso que implica, ha provocado un crecimiento de la demanda de educación. Por lo demás, toda la sociedad ha internalizado la necesidad de desarrollar la formación para quedar incorporados en la nueva realidad.

El rol social de una universidad culmina, entonces, cuando ésta es capaz de transmitir el saber. En la actualidad la demanda de conocimiento es particularmente relevante; a través del saber los miembros de una sociedad logran no sólo un mayor desarrollo personal, sino también llegar a ser elementos útiles para esta sociedad. En este sentido, las universidades privadas han cumplido un papel social de gran trascendencia, puesto que han permitido el acceso a sus aulas de un numeroso contingente de estudiantes que, de otro modo, hubiera quedado marginado de la posibilidad de aumentar sus conocimientos, de insertarse en el mundo actual y, en definitiva, de superarse como personas.

En suma, la resolución sobre la oferta de carreras está estrechamente vinculada a la misión que tiene toda universidad de educar. Al respecto, concordamos con Hernán Larraín cuando sostiene que: "pensar en educar a un hombre es sinónimo de un proceso que 
procura despertar en el educando todo el potencial que hay en él y en virtud del cual éste puede ser más persona. Se trata de suscitar el desarrollo autónomo de un individuo, en un proyecto que incluye lo intelectual, lo espiritual o moral, y lo físico, esto es, las diversas facetas del hombre" 4 .

Llama la atención que, en las encuestas sobre la materia que realizan empresas especializadas, sea frecuente encontrar un alto porcentaje de padres que aspira a que sus hijos tengan educación universitaria. Es probable que haya algún deseo de promoción social, pero también es indudable que este hecho refleja una demanda por instrucción universitaria, por considerarla un medio para lograr un mayor desarrollo económico, social, cultural y laboral.

Esta tendencia despierta cierto temor, pues podría constituir una fuente de frustración desde una doble perspectiva: por una parte, por muy alta que sea la oferta, no va a poder satisfacer toda la demanda; por otra parte, el sólo hecho de tener formación universitaria no garantiza éxito profesional o de otra índole. Sin embargo, otros la vemos con optimismo, porque para las universidades es un desafío ser capaces de preparar y ofrecer carreras atractivas, que habiliten a sus estudiantes para enfrentar los múltiples y variados escenarios que les ofrece el mundo moderno.

Si bien la carrera universitaria no es la única fuente de progreso, constituye una alternativa de educación superior que, bien estructurada, le dará al educando las herramientas que le abran sus expectativas de superación y progreso. Hoy en día, una carrera universitaria debe estructurarse de manera tal que no se reduzca a dar una restringida preparación profesional, sino que también entregue un bagaje cultural e intelectual que permita a su egresado una permanente evolución y adaptación al progreso del mundo moderno.

En consecuencia, en la toma de decisión sobre la oferta de carreras, la universidad está contribuyendo a todo el esfuerzo educativo de una colectividad entera, puesto que un país sin educación quedará rezagado en la dinámica de la supervivencia en este siglo que comienza.

4. Véase bibliografía final. 


\section{LAS UNIVERSIDADES PRIVADAS Y SUS PROYECTOS EDUCACIONALES}

Cada universidad privada tiene un proyecto de desarrollo institucional propio y en el marco de este proyecto debe resolver las grandes cuestiones que le afectan, y, en especial, tomar las decisiones sobre la oferta de carreras universitarias. Un proyecto institucional constituye un marco de referencia que en lo global indica los campos de acción que una universidad debe adoptar; no obstante, este marco no puede ser una estructura rígida porque la dinámica de nuestro mundo y la necesaria inserción de la universidad en el mismo, le exigen adecuarse a las realidades de la colectividad. Por lo tanto, la universidad deberá estar en condiciones de plasmar su proyecto institucional en forma tal, que sin apartarse de su misión, pueda ser protagonista del desarrollo de la sociedad en la cual participa.

No obstante esta amplitud, teóricamente no sería concebible que una universidad pretendiera incursionar, mediante la oferta de carreras, en áreas del conocimiento y del saber ajenas a su proyecto institucional.

\section{ESTRUCTURAR LA OFERTA DE CARRERAS}

Cuando una universidad decide abordar un determinado campo de carreras universitarias, debe considerar varios factores para hacer una evaluación.

El primero de ellos es determinar la contribución que puede hacer a la sociedad, es decir, si la o las carreras a impartir son necesarias y útiles a ésta y si están dentro de su natural esfera de actividades académicas. En seguida deberá considerar la posibilidad real de impartir dicha carrera y las perspectivas de su financiamiento. Para estos efectos, hay que evaluar los requisitos necesarios para impartir esa carrera. El punto de partida es determinar qué se quiere ofrecer realmente, lo que implica estructurar el programa ideal, del cual van a derivar las necesidades básicas en materia de infraestructura física, de elementos materiales como laboratorios, equipos computacionales, y las necesidades bibliográficas. Luego, hay que definir la estructura docente y estudiar la posibilidad de contar con autoridades y profesores idóneos. 
Estas consideraciones permiten establecer los costos operacionales del proyecto, a lo cual debe agregarse la estimación sobre el número ideal de alumnos a partir del conocimiento efectivo de la demanda potencial por el tipo de estudios que se piensa ofrecer.

Con base en este planteamiento y en este análisis académico y económico, se podrán determinar los recursos requeridos y su posibilidad de financiamiento, punto básico de inflexión para tomar la decisión de ofrecer o no la carrera.

El aspecto económico no puede ser despreciado y tampoco se puede omitir el tema de la oferta y demanda de una determinada carrera universitaria. En el análisis económico hay muchas variables a determinar, como la infraestructura física (inmuebles y equipamiento), las tecnologías a utilizar y su permanente actualización, las remuneraciones de los docentes, los gastos constantes en materia de laboratorios y servicios anexos, el valor de la colegiatura, etc., todas las cuales van a justificar el número de alumnos con el cual es viable una carrera.

Sin embargo, para tomar una decisión final no es absolutamente necesario que la nueva carrera deba autofinanciarse o generar excedentes, puesto que probablemente la universidad tenga convicciones y vocación propia para hacer una contribución a la sociedad, ofreciendo una carrera independientemente de las condiciones económicas o financieras.

Un ejemplo de lo anterior es la decisión de la Universidad Finis Terrae, de impartir Licenciatura en Historia, y eventualmente las posibles modalidades que ofrezca para carreras en el área de Educación.

Por lo tanto, considerando todas las variables que se han indicado, es la universidad la que en definitiva, en forma soberana y autónoma, debe resolver si ofrece o no una determinada carrera.

En resumen, para adoptar la decisión final deberá tener en cuenta numerosos elementos, el primero de los cuales será siempre si el proyecto en cuestión responde a su misión de universidad, y lo que esta institución quiere ofrecer a la sociedad. 
Luego tendrá que resolver sobre si va poder contar con los recursos humanos suficientes (directivos adecuados y con la necesaria experiencia y docentes idóneos), estructurar un plan de estudios con una malla curricular apropiada, que contemple las distintas asignaturas, sus requisitos, etc., contar con recursos bibliográficos que permitan respaldar los estudios y con recursos técnicos pedagógicos, de equipamiento y de infraestructura física pertinentes.

Teniendo en cuenta todos estos aspectos, si la universidad está interesada en ofrecer la carrera, si se encuentra capacitada para hacerlo y si cree que tendrá la posibilidad de captar al alumnado cuyo perfil también ha diseñado, debe ser soberana para tomar la decisión y lanzarse en esta riesgosa aventura de comenzar con una carrera universitaria. Digo "aventura" en el sentido de que siempre hay un riesgo, que la universidad deberá asumir seriamente a sabiendas de que una vez iniciada una carrera, su responsabilidad será ineludible frente a los futuros estudiantes, a sus padres y, en definitiva, ante toda la sociedad.

Comoquiera que sea, no son suficientes los campus, la infraestructura física, los laboratorios, los computadores, las bibliotecas, los convenios internacionales, las facilities, si la universidad no es capaz de desarrollar un programa que enseñe a pensar, a dialogar, a conocer la diversidad, a innovar, a buscar la verdad, a desterrar los prejuicios, etcétera. Es decir, la universidad no sólo deberá informar, sino también enseñar a pensar, para así desarrollar las potencialidades del educando a fin de que éste sepa adaptarse a las nuevas realidades y pueda enfrentarlas exitosamente.

\section{REGULAR LA OFERTA DE CARRERAS}

Cuando se habla de regular la oferta de carreras universitarias, la cuestión puede enfocarse desde dos puntos de vista distintos. El primero es preguntarse si una autoridad pública o privada, o un ente de cualquier naturaleza, pudiera determinar el número de carreras profesionales a ofrecer y el número de vacantes que debiera tener cada universidad o entidad. El segundo está relacionado con ciertas condiciones mínimas exigibles a las universidades, independientemente del número de carreras o de la posibilidad de ofrecer una en particular. 
Los planteamientos expuestos a lo largo de este trabajo permiten deducir nuestra respuesta para ambas preguntas. En efecto, tomando en consideración argumentos tales como: 1) la libertad de enseñanza; 2) la autonomía de las universidades y 3) la responsabilidad de los institutos educacionales, nuestra respuesta para ambas cuestiones es indudablemente negativa. Es decir, no podemos reconocer ni validar el que exista un ente, estatal o privado, dependiente o autónomo, que pudiera establecer o regular cuantitativa o cualitativamente la oferta de carreras universitarias. Cualquier intento de regulación, en especial cuantitativa, implica aceptar supuestos de ingeniería social o ingeniería cultural, que son totalmente descartables.

Hay elementos adicionales que sirven para refutar cualquier pretensión en este sentido:

a) ¿Por qué alguien se va a erigir en autoridad para determinar qué es lo que quiere o debe estudiar una persona que desee mayor educación, ampliar su formación, estar en condiciones más idóneas para participar del mundo contemporáneo?

b) ¿Con qué justificación se podría imponer que algunos accedan a una educación determinada o a una carrera específica y que otros queden excluidos, porque se han agotado los cupos?

c) ¿Quién es capaz de predecir qué va a requerir la sociedad en materia de profesionales en un futuro? Cinco, seis o siete años, plazo de duración normal de una carrera universitaria, es un tiempo demasiado amplio frente a la velocidad de los cambios tecnológicos en nuestro mundo global, para predecir si una carrera que hoy comienza va a ser necesaria o superflua de acuerdo a los requerimientos de la sociedad al final de ese período.

Cuántos errores pudieran haberse cometido en Chile si, por ejemplo, en los años 60 y 70 se hubiera descartado la carrera de Agronomía, a raíz de los avatares que padeció la agricultura, la que sin embargo, resurgió con gran potencia generando el cambio actual en materia de fruticultura, enología y otras actividades. Del mismo modo, ¿Quién puede predecir las necesidades de ciertas carreras tecnológicas, o del área de la salud, de las ciencias sociales o la psicología, etcétera? 
De tarde en tarde surgen aprensiones cuando aparecen nuevas carreras, como ha sucedido recientemente con la creación de facultades de Medicina en algunas universidades privadas.

En otras ocasiones se habla de la proliferación de determinadas carreras. Normalmente estas críticas emanan de intereses de corporaciones profesionales que quisieran mantener sus actuales ventajas derivadas de la escasa competencia y que ven amenazada su situación ante la eventualidad de que emerjan nuevas generaciones de colegas. Es el caso de las carreras de Medicina: mientras se critica la aparición de nuevas facultades que la imparten, la prensa denuncia el déficit de médicos en nuestro país. ¿Son ambas posturas consecuentes?

Del mismo modo, pretender imponer determinados requisitos para impartir una carrera, llámese años de estudios, un curriculum determinado $\mathrm{u}$ otros requerimientos, atenta contra la libertad de enseñanza y contra la decisión soberana de un establecimiento universitario. Será responsabilidad de cada institución determinar si está en condiciones de ofrecer una carrera y cuáles serán las formas en que impartirá la enseñanza respectiva, y será el mercado, a través de la capacidad para captar alumnos y/o posteriormente de la apreciación que se tenga sobre la calidad de sus profesionales, el que determine si realmente esa universidad será exitosa en el desarrollo de la carrera que imparta.

En definitiva, será la sociedad a través de los diversos mecanismos de que ella naturalmente dispone, la que estará en condiciones de apreciar la mayor o menor bondad de un proyecto educativo, la mayor o menor seriedad del establecimiento universitario, el mayor o menor prestigio de que éste goce en la comunidad, y de esa forma será la propia sociedad la que validará o no la oferta que de una o más carreras haga un establecimiento educacional.

Coincidiendo en este sentido con el pensamiento de Alvaro Bardón, no podemos afirmar que conocemos el futuro y que, por lo tanto, resulta fácil programar o planificar las demandas culturales y profesionales, sobre todo en el campo tecnológico y de los gustos individuales. Como señala Bardón, "la pretensión intelectual de 
modelar lo anterior conduce a esquemas rígidos y parciales, que se hacen inútiles o disfuncionales con rapidez. Lo mismo ocurre con la imposición de técnicas de enseñanza o programas de estudio y tantas modalidades de constructivismo cultural que sólo conducen a la obsolescencia rápida en una sociedad donde el cambio es la regla". ${ }^{5}$

\section{EL FUTURO DE LA OFERTA DE CARRERAS: LA INFORMACIÓN}

Mirando hacia delante, creemos que las universidades privadas, cada una con sus modalidades y características propias, serán las que deberán diseñar su propia oferta de carreras universitarias. De esta manera se estimulará una mejor calidad de la oferta, tanto desde el punto de vista de los docentes con que se vaya a contar, de los programas a desarrollar, de los métodos a utilizar, como de la infraestructura y de los elementos de apoyo de la misma.

Serán beneficiadas de esta manera aquellas instituciones que aparezcan como más sólidas, o con mejores proyectos, o bien aquellas que sepan posicionarse en los distintos nichos de la actividad educativa que ofrece la realidad cultural.

El gran desafío para las universidades privadas en la creación de carreras será que éstas incorporen las innovaciones metodológicas necesarias y la preparación adecuada de los futuros profesionales para enfrentar el mundo del siglo XXI, mediante competencias de las que carecen los actuales profesionales.

Para concluir, pensamos que existe un mecanismo mucho más objetivo y mucho más apegado a la realidad para regular la oferta de carreras. Este mecanismo no es otro que la información que se ofrezca a la comunidad respecto de todo el quehacer universitario.

La información deben proporcionarla en primer lugar las propias instituciones educacionales; en segundo término, ojalá entidades independientes y especializadas, capacitadas para recoger, de cada una

5. Véase bibliografía final. 
de las instituciones aspectos que sean relevantes, objetivos, comparables y que sirvan de base para análisis cualitativos, todos los cuales estarán destinados a aportar la información necesaria para que quien deba adoptar una decisión, pueda evaluar y resolver de la manera más razonada posible.

Sólo la información hará posible que el requirente de una determinada carrera pueda tomar una decisión independiente. Esto supone que junto con garantizar una información objetiva, se pueda sancionar toda divulgación torcida, distorsionada o no acorde con la realidad.

Si la colectividad logra constituir un circuito de información que garantice la aplicación de parámetros de objetividad y de comparación sobre elementos semejantes, estará a su vez garantizando que la oferta sea razonable y adecuada a los requerimientos de la sociedad.

\section{RefERENCiAS Bibliográficas y Notas}

1. LOCE (Ley Orgánica Constitucional de Enseñanza, 1990), № 18.962, art. 75 .

2. LOCE (Ley Orgánica Constitucional de Enseñanza, 1990), № 18.962, art. 76

3. Esta afimación no significa que toda universidad deba necesariamente hacer investigación. Al efecto, véase, entre otros artículos del mismo autor: Brünner, José Joaquín, "Las universidades privadas y el reto de la innovación"; en Revista Finis Terrae N4, Chile, 1996, p.83.

4. Larraín Fernández, Hernán, "La educación en el futuro de Chile"; en Economía y Sociedad N 64, Chile, 1987, p.20.

5. Bardón, Alvaro, "Educación superior: una crítica"; en Revista Finis Terrae No 9, Chile, 2001, p.15. 